\title{
15 YEARS REVIEW OF GASTROCNEMIUS FLAP DONE IN DEPARTMENT OF ORTHOPAEDICS, HOSPITAL TENGKU AMPUA AFZAN, KUANTAN
}

\author{
Nazri bin Mohd Yusof ${ }^{1}$ \\ ${ }^{1}$ Department of Orthopaedic, Traumatology and Rehabilitation, Kulliyyah of Medicine, \\ International Islamic University of Malaysia, Kuantan, Pahang, Malaysia.
}

Presenter: Nazri bin Mohd Yusof, nazrimy2000@gmail.com

Introduction: Gastrocnemius flap is the workhorse for wound coverage in the proximal tibia. It can be perform by general orthopaedic surgeon because it is done without the need of microscopic instrumentation. Its coverage can be extended to cover the knee and midshaft of tibia when skin overlying it is included in the flap.

Materials and method: From July 2002 till January 2017, 47 patients underwent gastrocnemius flap in our hospital ( 26 muscular and 21 musculocutaneous flap). 41 flaps were raised from medial, 5 lateral and 1 from both muscle belly. There were 41 males and 6 females with the mean age of 21.4 years old (range 13-82). The flaps were done to cover upper third (29), upper half (6), midshaft tibia (6), knee (2) and distal femur (1). The initial problems were open fracture (30), infection following plating (9), necrotising fasciitis (4), degloving injury (2), pin site infection (1) and melioidosis (1). Eight patients have underlying Diabetes mellitus, 2 Hepatitis B, 3 HIV or 1 COAD. Two patients have sciatic nerve palsy.

Results: There was no flap necrosis. Complications include persistence infection in 6 patients which require repeated debridement (5), below knee amputation (1), advancement of flap (1) and additional fasciocutaneous flap (1). Two patients with gastrocnemius musculocutaneous flap had partial failure of skin graft that heals with dressing. One patient develops transient peroneal nerve palsy following the lateral gastrocnemius transfer.

Conclusion: Gastrocnemius flap is a reliable and safe procedure in orthopaedic surgery. Problems that occur are associated with inadequate debridement and underestimate the size of flap required. 